\title{
Objektrekonstruktion in der Mikrowellen-Defektoskopie
}

\author{
Thomas Gagelmann ${ }^{1}$, Maxim Nesterov'1, Sebastian Wöckel', \\ Cuihua Li², Sebastian Hantscher', Jörg Auge ${ }^{2}$ \\ ${ }^{1}$ Institut für Automation und Kommunikation, Werner-Heisenberg-Str. 1, \\ 39106 Magdeburg, Deutschland \\ ${ }^{2}$ Hochschule Magdeburg-Stendal, Breitscheidstr. 2, 39114 Magdeburg, Deutschland
}

\section{Zusammenfassung}

Dieser Beitrag stellt die Ergebnisse eines geförderten Projekts vor, dessen Ziel es war, die erreichbare Auflösung bei der Mikrowellen-Defektoskopie mit niedrigen Prüffrequenzen zu verbessern. Bei niedrigen Prüffrequenzen, wie die im Projekt verwendeten $24 \mathrm{GHz}$, wird eine größere Eindringtiefe der Mikrowellen ins Material erreicht. Es treten aber auch vermehrt Beugungseffekte auf, die die erreichbare Ortsauflösung verschlechtern und die Klassifizierung von Defekten erschweren.

Der im Artikel vorgestellte Ansatz verwendet eine der Messung nachgelagerte Analysetechnik, bei der die Messdaten mit einer zuvor ermittelten Transferfunktion entfaltet werden, um so die Beugungseffekte zu unterdrücken. Es wird gezeigt, dass mit dieser Technik für bestimmte Anwendungsfälle die Beugungsgrenze bei der Abbildung von kleinen, eng bei einander liegenden Defekten überwunden werden kann. Außerdem werden Defektkanten schärfer dargestellt, was eine genauere Klassifizierung der Defekte ermöglicht.

Keywords: zerstörungsfreie Werkstoffprüfung, Mikrowellen-Defektoskopie, Rekonstruktion, Punktspreizfunktion, Entfaltung

\section{Einleitung}

Der Markt für glasfaserverstärkte Kunststoffe wächst in Deutschland überdurchschnittlich stark. Deswegen werden kostengünstige und robuste zerstörungsfreie Prüfmethoden für diese Art dielektrischer Verbundmaterialien immer wichtiger um eine gleichbleibend gute Qualität und effiziente Produktion sicherzustellen. Die aktuell kommerziell verfügbaren zerstörungsfreien Prüfmethoden sind für glasfaserverstärkte Kunststoffe allerdings oft nur eingeschränkt geeignet. Zum Beispiel ist die Eindringtiefe bei der Thermografie auf wenige Millimeter begrenzt. Bei akustischen Prüfverfahren wird das Signal stark gedämpft und es treten Streueffekte an dem hohen Faseranteil im Material auf [1, 2]. Radiologische Prüfverfahren sind wiederum teuer, es werden zusätzliche Strahlenschutzvorkehrungen benötigt und die maximale Größe der Prüfobjekte ist oft begrenzt [3, 4]. Eine andere Methode zur Prüfung glasfaserverstärkter Kunststoffe, ist die MikrowellenDefektoskopie. Mit Ihr können zerstörungsfrei Defekte im Probekörper bildhaft dargestellt und klassifiziert werden. Die Wahl der Prüffrequenz hat dabei einen entscheidenden Einfluss auf die Eindringtiefe und die erreichbare Auflösung. Bei niedrigen Prüffrequenzen treten außerdem verstärkt Beugungserscheinungen an Inhomogenitäten im Material (Ecken, Kanten, Defekte) auf. In der Praxis stellen diese Beugungserscheinungen ein Problem dar, da sie insbesondere Defekte nahe von Ecken und Kanten maskieren können und die Klassifizierung der Defekte bezüglich Ihrer Größe Form und Tiefenlage erschweren [5-9]. Das bedeutet für die Qualitätskontrolle eine deutlich verringerte Zuverlässigkeit der Detektion von randnahen gegenüber randfernen Fehlern.

In der $\mathrm{THz}$-Technik werden diese Probleme durch die Verwendung vergleichsweise hoher Prüffrequenzen umgangen. Bei dieser Technik treten Beugungsmuster von Ecken und Kanten im Vergleich zu niedrigen Frequenzen nur in abgeschwächter Form auf, dafür ist sie aufgrund des erhöhten technischen Aufwands allerdings auch immer noch recht teuer und die Eindringtiefe der hochfrequenten Mikrowellen in das Material ist aufgrund der starken Dämpfung begrenzt [10-13].

In diesem Zusammenhang bietet sich für die Detektion tiefliegender Defekte die Verwendung von niedrigeren Prüffrequenzen an. Im Projekt wurde eine Prüffrequenz von $24 \mathrm{GHz}$ verwendet. Mit dieser Prüffrequenz können mit relativ geringem technischen Aufwand und einer geringen Sendeleistung größere Eindringtiefen in das dielektrische Material erzielt werden.

Der im Projekt verwendete Messaufbau ist in Abbildung 1 dargestellt. Bei einer Messung 
bewegt sich die Hohlleiterantenne im gleichbleibenden Abstand von $4 \mathrm{~mm}$ zeilenweise über die Oberfläche des Prüfobjekts, bestrahlt es mit Mikrowellen einer konstanten Frequenz von $24 \mathrm{GHz}(\mathrm{CW})$ und zeichnet den komplexen Reflexionsparameter $S_{11}$ auf. Das Messsignal enthält das Interferenzmuster der Wellen, die an den Inhomogenitäten innerhalb des Probekörpers gestreut bzw. gebeugt wurden.
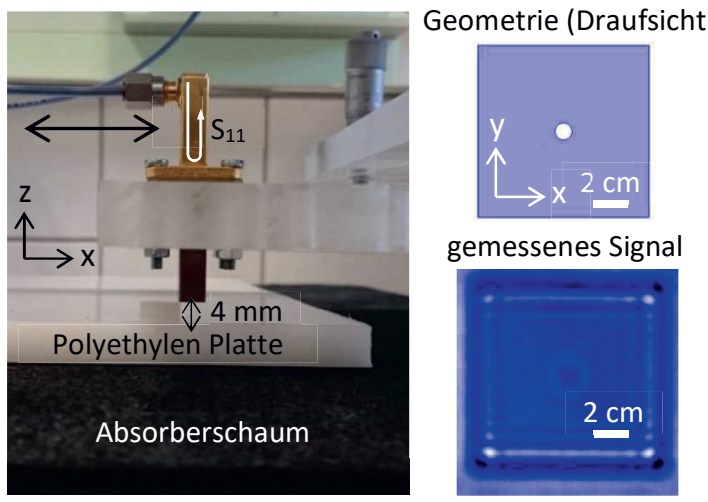

Abb. 1: Experimentelle Messanordnung mit der Hohlleiterantenne über dem Probekörper (links), ein Schema eines Probekörpers mit einer einzelnen Lochbohrung (oben rechts), und dem gemessenen Reflexionssignal $\left|S_{11}\right|$ (unten rechts).

In Abbildung 2 ist das Problem der durch die Beugungsmuster maskierten Defekte anhand einer Messung dargestellt, bei der vor allem der Defekt im rot markierten Bereich nur schwer erkennbar ist. Das Ziel des hier vorgestellten Projekts war es, eine Methode zu entwickeln, die trotz vorhandener Beugungserscheinungen eine klare Identifikation und Klassifikation von Defekten ermöglicht, deren Abmessungen klein gegenüber der Wellenlänge sind. Dafür müssen die Beugungseffekte möglichst unterdrückt werden, um so die Kantenschärfe der dargestellten Objekte zu erhöhen und sie in der korrekten Größe abzubilden. Dazu bedarf es einer Verfeinerung der Aufzeichnungstechnik, der Modellierung der Antenne sowie einer Bildverarbeitung bei der eine der Messung nachgelagerte Objektrekonstruktion durchgeführt wird um die Beugungsmuster zu reduzieren. Bei der Rekonstruktion wird davon ausgegangen, dass das gemessene Reflexionsprofil mit den Beugungsmustern $h$ mathematisch der mit GI. (1) beschriebenen Faltung des geometrischen Profils des Probekörpers $f$ mit einer zunächst unbekannten Punktspreizfunktion (PSF) $g$ entspricht, wobei mit $*$ der Faltungsoperator bezeichnet wird.

$$
h=f * g
$$

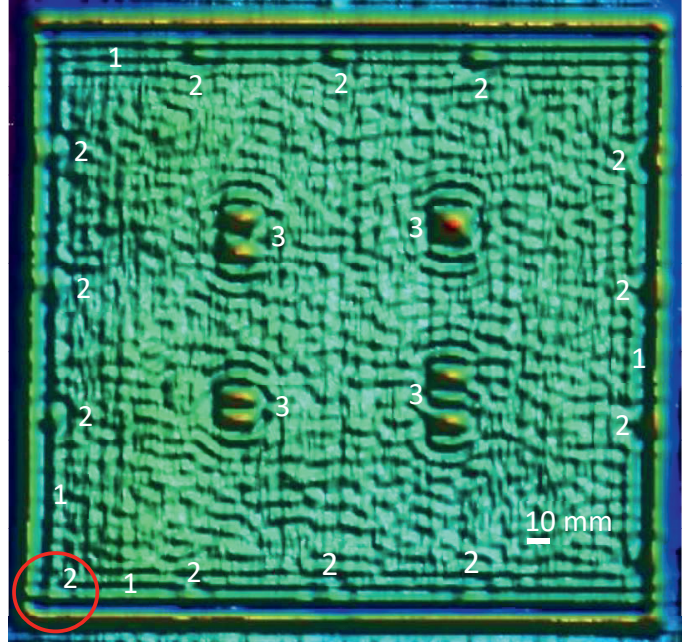

Abb. 2: Dargestellt ist der Realteil des Reflexionsparameters $S_{11}$ vom 2DScan eines Probekörpers mit den an Geometriesprüngen entstehenden Beugungserscheinungen. Im Bild sind die verschiedenen Beugungserscheinungen mit Zahlen markiert. 1) Beugungseffekte an den Kanten des Probekörpers aus Polyethylen,

2) Beugungseffekte an mit Luft gefüllten Bohrungen von $3 \mathrm{~mm}$ Durchmesser 3) Beugungseffekte an mit Zylindern aus Aluminium gefüllten Bohrungen von $3 \mathrm{~mm}$ Durchmesser.

Zur Bestimmung der Objektkontur aus den Messdaten ist eine Entfaltung notwendig. Diese Entfaltungsmethode wird in der Optik, zum Beispiel in der Mikroskopie, bereits erfolgreich eingesetzt, um die Auflösung und Bildqualität zu verbessern [14]. In der Optik hängen die Eigenschaften und Qualität eines Bildes maßgeblich vom Fokus und dem Linsensystem des optischen Messsystems selbst und weniger vom abgebildeten Objekt ab. In der Mikrowellen-Defektoskopie hat aber neben dem Messsystem auch die Beschaffenheit des Prüflings einen entscheidenden Einfluss auf die abgebildeten Beugungsmuster, da sich der Prüfling im Nahfeld der Antenne befindet um eine hohe Auflösung zu erzielen. Deswegen wird im Projekt überprüft, inwiefern dieses Modell auch für die Mikrowellen-Defektoskopie anwendbar ist und welche Vor- und Nachteile sowie Limitationen bei der Entfaltungsmethode auftreten.

Die Punktspreizfunktion $g$ ist definiert als die Antwort des Messsystems auf ein ideales infitesimal kleines punktförmiges Objekt. Die Faltung wird aus Gründen der einfacheren Berechnung im Frequenzbereich durch die Multiplikation der Fouriertransformierten von $f$ und $g$ berechnet. Die für die Entfaltung 
benötigte Umkehroperation ist dann die in GI. (2) dargestellte einfache Division, wobei die Fouriertransformierten der jeweiligen Funktionen in Großbuchstaben angegeben sind.

$$
F=\frac{H}{G}
$$

Die Fouriertransformierte des rekonstruierten geometrischen Profils $F$ wird nach der Entfaltung durch die Anwendung einer inversen Fourier-Transformation zurück in den Zeitbereich überführt. Sofern die Punktspreizfunktion $g$ bekannt ist, kann das geometrische Profil des Probekörpers $f$ aus dem gemessenen Signal $h$ mithilfe der Entfaltungsoperation rekonstruiert werden. Auf diese Weise werden die abgebildeten Beugungseffekte an Ecken und Kanten von Objekten nachträglich unterdrückt. In Abbildung 3 wird das Ergebnis einer solchen Entfaltung anhand synthetischer Daten dargestellt.

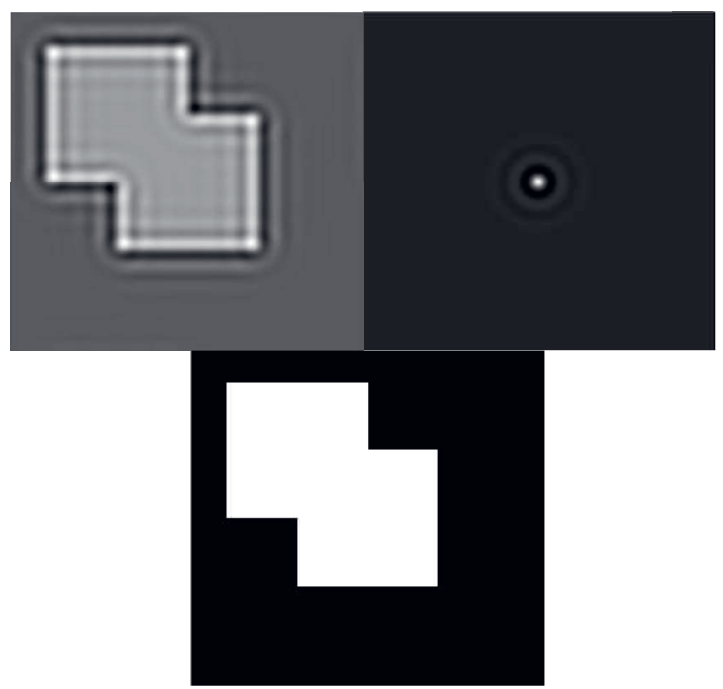

Abb. 3: Darstellung der Unterdrückung von Beugungserscheinungen anhand synthetischer Daten. Das Objekt mit Beugungserscheinungen $h$ (links), die Transferfunktion bzw. PSF $g$ (rechts) und das rekonstruierte Objekt $f$ (unten).

\section{Methodik}

Es können zwei Hauptansätze verfolgt werden, um die Bildauflösung zu erhöhen, die Beugungseffekte zu reduzieren und die Defektklassifizierung in der Mikrowellen-Defektoskopie zu verbessern. Erstens kann die Messtechnik in Bezug auf ihre Emissionseigenschaften wie Strahlfokussierung, Rückflussdämpfung und Größe der Antennenfußfläche verbessert werden. Die Ergebnisse einer solchen Antennenoptimierungsstudie wurden in früheren Arbeiten vorgestellt [15].
Der aktuelle Beitrag zeigt die Ergebnisse eines zweiten Ansatzes, bei dem die Datenanalysetechniken in der Nachverarbeitung eingesetzt werden. Konzeptionell ist der Datenanalyseansatz in zwei Phasen unterteilt. Die erste Phase entspricht dem Training von Algorithmen, bei dem verschiedene Messszenarien numerisch simuliert und zur Bestimmung der spezifischen Punktspreizfunktionen verwendet werden. Die numerische PSF muss analytisch approximiert und eine Modellbibliothek erstellt werden, die die jeweiligen analytischen Punktspreizfunktionen für verschiedene Kombinationen von Antennen, Probenmaterial und Defekttypen enthält. In der zweiten Phase, der Anwendungsphase, werden die in der Modellbibliothek gespeicherten Punktspreizfunktionen unter realen Bedingungen verwendet, um das Beugungsmuster aus den gemessenen Bildern zu entfernen, wodurch klare und scharfe Objektprofile erzeugt werden. Nachdem die Bibliothek von Übertragungsfunktionen erstellt wurde, erfordert die Anwendungsphase keine großen Rechenressourcen mehr.

Der vorgeschlagene Arbeitsablauf kann mit den folgenden Schritten dargestellt werden.

Lernphase:

1. Numerische Simulation von PSFs

2. Analytische Näherung von PSFs

3. Erstellung der PSF-Bibliothek

Anwendungsphase:

4. Auswahl geeigneter PSF aus der Bibliothek

5. Entfaltung

6. Fehlerklassifizierung

Im ersten Schritt müssen für eine endliche Menge von Messszenarien PSF numerisch simuliert werden. Eine PSF ist dabei nur für ein bestimmtes, simuliertes Messszenario gültig. Um auch Punktspreizfunktionen für die große Menge an Messszenarien zu erhalten, die zwischen den simulierten Szenarien liegen (unterschiedliche Defektformen und -größen, Positionstiefe), werden die numerischen Punktspreizfunktionen im zweiten Schritt durch Anpassen geeigneter Funktionen analytisch approximiert. Jede Kombination von Antenne, Proben- und Defektmaterialien verfügt über eine eigene analytische PSF, die im Schritt 3 in einer Bibliothek zusammengefasst werden.

In der Anwendungsphase wird im Schritt 4 eine geeignete PSF aus der Bibliothek ausgewählt. Der Antennentyp und das Probenmaterial sind bei Messungen in der Regel bekannt. Der Defekttyp und das Defektmaterial müssen jedoch bestimmt werden. Dies kann durch Minimieren der Fehlerfunktion $\|h-f * g\|^{2}$ erreicht werden, wobei $h$ ein gemessenes Reflexionsprofil, $f$ ein unbekanntes Defektprofil und $g$ eine analytische PSF aus der Bibliothek 
darstellt. Die Minimierung wird für jede PSF aus der Bibliothek durchgeführt. Danach wird die PSF mit dem geringsten Minimierungsfehler für die Entfaltung ausgewählt. Die Entfaltung in Schritt 5 sollte dann das beugungsfreie Bild liefern, mit dessen Hilfe die Defekte in Schritt 6 klassifiziert werden können.

Im aktuellen Beitrag werden vor allem die Schritte 1 und 5 der vorgeschlagenen Datenanalyse basierend auf Simulationen, Messungen und empirischen Ergebnissen für ausgewählte Fehlerklassen erörtert.

\section{Diskussion}

Es wurde ein Satz von Probekörpern mit Defekten verschiedener Typen und Größen simuliert und gemessen. Danach wurde die auf Entfaltung basierende Rekonstruktionsmethode angewendet, um die Auflösung zu verbessern.

Für die Rekonstruktion wird eine zum Messszenario passende PSF benötigt. Die Generierung einer idealen Punktspreizfunktion aus Mess- oder Simulationsdaten ist allerdings schwierig, da die von einem infitesimal kleinem punktförmigem Objekt reflektierte Energie zu gering ist, um sie zu detektieren. Im Rahmen des Projekts wurden drei Ansätze getestet, mit denen zumindest eine gute Näherung der PSF aus dem Mess- bzw. Simulationsdaten generiert werden sollte.

Der erste Ansatz war die Anwendung der Entfaltung für ein Objekt, bei dem das ideale geometrische Profil bekannt war, so dass $f$ synthetisch nachgebildet werden konnte. Durch das Umstellen der GI. (2) sollte die Entfaltung von $G=H / F$ dann theoretisch die PSF $g$ liefern. Allerdings stellte sich bei den praktischen Versuchen mit Messdaten heraus, dass mit dieser Methode für unterschiedliche Objektgrößen unterschiedliche PSF erzeugt wurden. Für im Vergleich zur Wellenlänge kleine Objekte wurden physikalisch sinnvolle lokalisierte PSF mit ähnlichem Funktionsverlauf erzeugt. Bei größeren Objekten lieferte die Entfaltung allerdings keine lokalisierten Funktionen mehr. Die erzeugten Funktionen glichen eher einem symmetrischen Rauschen und stellten eine reine mathematische Umkehrfunktion ohne physikalische Relevanz dar. Dieses Ergebnis ist ein erster Hinweis darauf, dass das aus der Optik übertragene Modell in der Mikrowellen-Defektoskopie nur eingeschränkt gültig ist.

Die zweite getestete Methode, eine PSF aus den Messdaten zu extrahieren, bestand darin, statt einem Punktdefekt eine scharfe Objektkante zu vermessen, um auf diese Weise die Kantenspreizfunktion (ESF) zu erhalten. Durch Ableitung der ESF orthogonal zum
Kantenverlauf kann die Linenspreizfunktion (LSF) berechnet werden, die dem Querschnitt durch die dreidimensionale PSF entspricht [16]. Mithilfe dieser Methode konnten lokalisierte PSF erzeugt werden, die allerdings keine Spiegelsymmetrie entlang der y-Achse aufwiesen, wie sie für PSF eigentlich typisch ist. Bei der Entfaltung lieferten sie aber trotzdem ähnlich gute Ergebnisse, wie die mit der dritten Methode erzeugten PSF.

Die dritte Methode bestand darin, den kleinstmöglichen, noch messbaren Defekt zu vermessen und dessen Reflexionsprofil als PSF zu nutzen. So kann die für die Entfaltung benötigte PSF einfach und in guter Näherung erzeugt werden.

Aufgrund der einfacheren Umsetzung und dem symmetrischeren Funktionsverlauf wurde hauptsächlich auf diese dritte Methode zur Erzeugung der PSF zurückgegriffen. Der mit dem in Abbildung 1 dargestellten Messsystem kleinste messbare Defekt war ein zylindrischer Defekt aus Aluminium von $3 \mathrm{~mm}$ Durchmesser, der in eine $10 \mathrm{~mm}$ dicke Platte aus Polyethylen eingelassen war. Der Realteil des 2D-Scan dieses kleinen Referenzdefekts ist in Abbildung 5 oben links zu sehen.

Zur Vereinfachung der Bildanalyse wurden aus den jeweiligen 2D-Scans eindimensionale Schnittprofile, die mittig durch die Defekte verlaufen, extrahiert. Die Schnittebenen sind mit gestrichelten Linien angedeutet. Mit diesen Schnittprofilen wurde die Entfaltung durchgeführt.

In der Praxis sind die Messsignale in der Regel mit einem additiven Rauschen behaftet. Dieses Rauschen wird bei der Entfaltung mittels einer einfachen Division vor allem für die hohen Frequenzanteile im Messbild überproportional verstärkt. Um das zu vermeiden, wurde die Entfaltung mithilfe des Wiener-Filters umgesetzt $[17,18]$. Die Aufgabe des Wiener-Filters ist es, die gestörten hohen Frequenzanteile aus den Messdaten herauszufiltern. Für den Frequenzbereich wird er mit GI. (3) beschrieben.

$$
F=\left[\frac{G^{*}}{|G|^{2}+K}\right] \cdot H
$$

Dabei ist $G^{*}$ die komplex Konjungierte von $G$ und die Konstante $K$ repräsentiert eine Abschätzung des Verhältnisses von Rauschzur Signalleistung. Für niedrige Frequenzen entspricht der Wiener-Filter der in der Einleitung vorgestellten einfachen Division. Hohe Frequenzen werden jedoch herausgefiltert, wobei die Bandbreite des Filters durch die Wahl von $K$ bestimmt wird.

Um die Frage zu beantworten, inwiefern die Qualität der Messungen durch die Rekonstruktionsmethode verbessert werden kann, soll 
zunächst die Rekonstruktion eines im Vergleich zur Wellenlänge großen Defekts betrachtet werden. Abbildung 4 zeigt die Ergebnisse einer Messung eines großen, L-förmigen Defekts aus Aluminium, eingebettet in einer $10 \mathrm{~mm}$ dicken Platte aus Polyethylen.
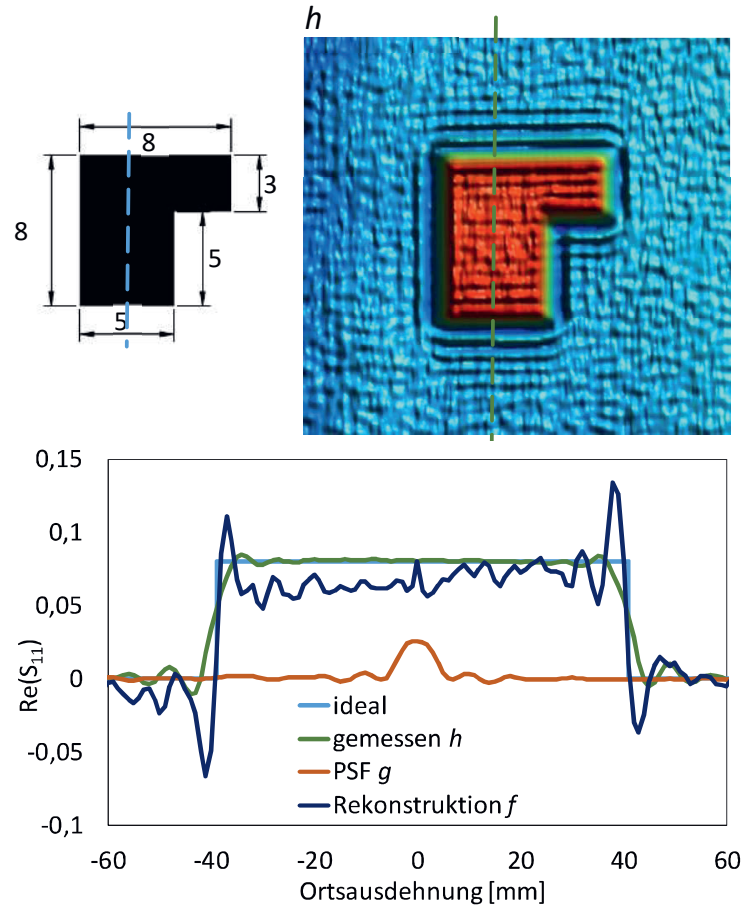

Abb. 4: Bildrekonstruktion für einen großen L-förmigen Defekt. Schematische Darstellung des Defekts (oben links), des Realteils des gemessenen Reflexionskoeffizienten $S_{11}$ als 2D-Scan für den metallischen L-förmigen Defekt (oben rechts). Darstellung der 1DSchnittprofile des gemessenen Defekts $h$, der PSF $g$, des rekonstruierten Defektprofils $f$ und des idealen Defektprofils (unten).

Die Abmessungen des Defekts sind im Schema links oben in Millimetern angegeben. Das gemessene Signal wird als komplexer Reflexionskoeffizient $S_{11}$ in einem 2D-Scan aufgezeichnet, dessen Realteil im Bild rechts oben dargestellt ist. In der unteren Grafik in Abbildung 4 sind die Schnittprofile der ursprünglichen Messung in grün und die Rekonstruktion des Defekts in dunkelblau dargestellt. Es ist erkennbar, dass der mit der Rekonstruktion einhergehende Informationsverlust Artefakte im Defektprofil erzeugt. Dadurch wird die Bildqualität insgesamt gesehen eher verschlechtert. Eine Verwendung des Rekonstruktionsalgorithmus zur Verbesserung der allgemeinen Bildqualität ist daher fraglich. Andererseits konnte die Kantenschärfe am Übergang vom Polyethylen zum Aluminium mit der Rekonstruktion deutlich verbessert werden.
Die Objektflanken folgen nach der Rekonstruktion dem idealen Profil sehr genau, was die Größenklassifizierung des Defekts vereinfacht.

Ein weiterer interessanter Anwendungsfall ist die Rekonstruktion von im Vergleich zur Wellenlänge kleinen Defekten. Die Wellenlänge bei $24 \mathrm{GHz}$ in Luft beträgt ca. $12,5 \mathrm{~mm}$. Vor allem kleine Defekte können leicht von Beugungsmustern überlagert werden, was ihre Erkennung erschwert. Es wurden zwei identische, zylindrische Defekte aus Aluminium mit $3 \mathrm{~mm}$ Durchmesser vermessen, die in einer $10 \mathrm{~mm}$ dicken Platte aus Polyethylen eingelassen waren. Die Defekte lagen so nahe beieinander, dass sich die von ihnen erzeugten Beugungsmuster überlagerten. In Abbildung 5 ist oben rechts das Schema der Defekte und der Realteil des 2D-Scans dargestellt. Wegen der Überlagerung der Beugungsmuster sind die einzelnen Defekte nicht unterscheidbar. Sie erscheinen im Bild wie ein einzelner, etwas größerer Defekt. Für die Rekonstruktion wurde wieder das Defektprofil eines einzelnen, zylindrischen Defekts aus Aluminium mit $3 \mathrm{~mm}$ Durchmesser als PSF verwendet, dessen 2D-Scan in Abbildung 5 oben links zu sehen ist.
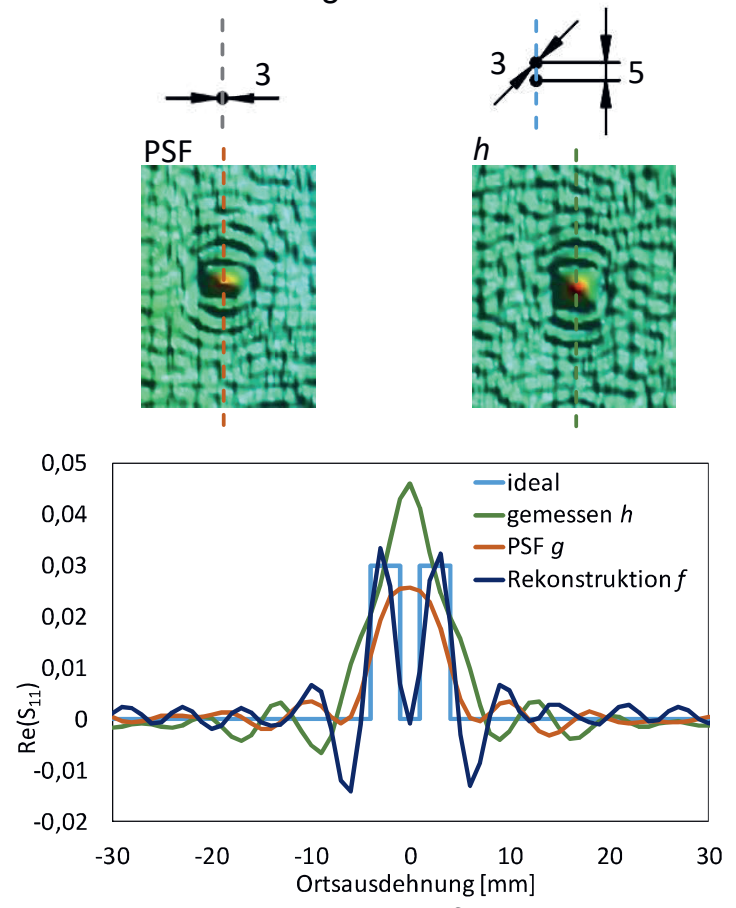

Abb. 5: Bildrekonstruktion für zwei kleine zylindrische Metalldefekte in unmittelbarer Nähe. Reflexions-koeffizient $S_{11}$ als 2D-Scan für einen einzelnen Defekt (oben links) und für zwei Defekte (oben rechts). Das gemessene $h$, die PSF $g$, das rekonstruierte Profil $f$ und das ideale geometrische Profil im Vergleich als 1D-Scan (unten). 
In der unteren Grafik in Abbildung 5 sind die Schnittprofile durch die Defekte dargestellt. Während in der in grün dargestellten Originalmessung nur ein einzelner, zentraler Peak zu sehen ist, lassen sich nach der Rekonstruktion zwei klar getrennte Peaks ausmachen, die dem idealen Defektprofil sehr genau folgen. Mit Hilfe der Rekonstruktion konnten die Defekte also separiert werden.

Die Rekonstruktion von sich überlagernden Beugungsmustern funktioniert allerdings nur für ähnliche Defekte, für deren Entfaltung die gleiche PSF verwendet werden kann. Bei Defekten aus unterschiedlichen Materialien, zum Beispiel ein metallischer Einschluss neben einer mit Luft gefüllten Bohrung, funktioniert die Entfaltung nicht, da für die Rekonstruktion der verschiedenen Defekte unterschiedliche PSF verwendet werden müssten. Das ist der Hauptunterschied in der Nutzung des Entfaltungsmodells in der Mikrowellen-Defektoskopie und der Optik, wo die PSF nur vom Optischen System, nicht aber vom Prüfobjekt abhängig ist. In der Mikrowellen-Defektoskopie ist die Intensität und Form der Beugungsmuster um ein Objekt von dessen Reflexionsfläche, dem Abstand der Reflexionsfläche zur Antenne und des Unterschieds der Permittivität von Defektund dem Defekt umgebenden Material abhängig. Dadurch entstehen Beugungsmuster von unterschiedlicher Intensität, wobei Beugungsmuster starker Intensität die Beugungsmuster mit schwächerer Intensität überlagern können.

\section{Zusammenfassung}

In diesem Artikel wurde ein neuartiger zweistufiger Ansatz für die Mikrowellen-Defektoskopie vorgestellt, um Beugungseffekte, die an Inhomogenitäten im Prüfobjekt entstehen, nachträglich aus den Messdaten zu entfernen. Die vorgestellte Methodik basiert auf numerischen Simulationen zur Erzeugung von geeigneten Punktspreizfunktionen in der Lernphase und einem aus der Optik bekannten Entfaltungsansatz für die Objektrekonstruktion in der Anwendungsphase.

Es wurden außerdem drei Methoden getestet, um eine für die Entfaltung geeignete Punktspreizfunktion aus den Simulations- bzw. Messdaten zu extrahieren. Die im Projekt bevorzugt verwendete Methode bestand darin das Reflexionsprofil des kleinsten, messbaren Defekts in der Entfaltung zu verwenden. Die praktische Umsetzung dieser Methode ist einfach und das gemessene Reflexionsprofil stellt eine gute Näherung der idealen PSF dar.

Bei der Objektrekonstruktion wurden die gemessenen Daten mit der vorab generierten PSF unter Verwendung des Wiener-Filters entfaltet. Diese Rekonstruktionsmethode wurde durch die experimentelle Vermessung von einfachen synthetischen Defekten und durch 3DFeldsimulationen verifiziert. Dabei konnte ein besseres Verständnis für das Potential, aber auch die Einschränkungen dieses Ansatzes in der Mikrowellen-Defektoskopie gewonnen werden. Es wurde gezeigt, dass mit der Entfaltungsmethode Beugungsmuster unterdrückt werden können, allerdings nur auf Kosten der allgemeinen Bildqualität, da durch den Informationsverlust bei der Entfaltung Artefakte entstehen. Die Defektgrenzen können mit gleichzeitiger Erzeugung von Artefakten, die eine Art Überschwingen an der Objektkontur darstellen, jedoch schärfer abgebildet werden. Wenn für die Defektklassifizierung die Darstellung der exakten Defektkonturen wichtiger ist, als die photographische Darstellung des Messbildes, kann das Rekonstruktionsverfahren hilfreich sein.

Die experimentellen Ergebnisse deuten außerdem an, dass zwei identische Defekte, die deutlich kleiner als die Wellenlänge des Messsignals sind und sehr nahe beieinander liegen, nach der Rekonstruktion einzeln aufgelöst werden können. Die klassische Beugungsgrenze des Messsystems kann in diesem speziellen Fall also überwunden werden. Auf den ersten Blick scheint die Beschränkung der Anwendbarkeit des Entfaltungssatzes bei der Überlagerung von Beugungsmustern auf identische Defekte eine starke Einschränkung zu sein. In der Praxis dominiert jedoch in einer großen Anzahl von Materialien meist eine Art von Defekt.

Die Experimente haben allerdings auch gezeigt, dass für jedes Messszenario in Abhängigkeit von Messsystem, Antenne, Material des Defekts und des den Defekt umgebenden Materials, der Größe der Reflexionsfläche und des Abstandes der Reflexionsfläche zur Antenne eine eigene PSF bestimmt werden muss. Das macht die Lernphase dieses Verfahrens sehr aufwändig und verhindert, dass es wie in der Optik als allgemeingültiges Werkzeug für ein bestimmtes Messsystem eingesetzt werden kann. Der im Artikel vorgeschlagene Ansatz für eine automatisierte Bildrekonstruktion und Defektklassifizierung, welche eine analytische Näherung einer geeigneten PSF verwendet, bietet Potential für einen begrenzten Satz von vorab definierten Messszenarien. Die große Menge von möglichen Messszenarien, verbunden mit dem hohen zeitlichen Aufwand der 2D-Simulationen bzw. Messungen zur Generierung geeigneter Punktspreizfunktionen beschränkt allerdings die praktische Anwendbarkeit des vorgestellten Ansatzes für allgemeinere Anwendungen. 


\section{Danksagung}

Die finanzielle Unterstützung für die beschriebene Arbeit im Rahmen des IGF-Projekts 18827 BG durch die Forschungsgemeinschaft "Fördergemeinschaft für das SKZ" über AiF im Rahmen des Programms zur Förderung kooperativer industrieller Forschung und Entwicklung (IGF) des Bundesministeriums für Wirtschaft und Energie (BMWi), die auf einer Entschließung des Deutschen Bundestages basiert, wird dankend anerkannt. Darüber hinaus möchten wir uns beim Europäischen Fonds für regionale Entwicklung (EFRE) bedanken, der diese Forschung durch die Finanzierung der Radarsensoren ermöglichte.

\section{Referenzen}

[1] R. S. Panda, O. Karpenko, L. Udpa, M. Haq, P. Rajagopal, K. Balasubramaniam, "Rapid noncontact inspection of composite ailerons using air-coupled ultrasound", AIP Conference Proceedings 1706, 080002 (2016)

[2] M. Webersen, S. Johannesmann, J. Düchting, L. Claes, B. Henning, "Guided ultrasonic waves for determining effective orthotropic material parame-ters of continuous-fiber reinforced thermoplastic plates", Ultrasonics 84, S. 53-62, 2017.

[3] A. du Plessis, S. Roux, A. Guelpa, "Case Studies in Nondestructive Testing and Evaluation”, vol. 6, Part A, Nov. 2016, pp 17-25

[4] L. De Chiffre, S. Carmignato, R. Kruth, J.P. Schmitt, A. Weckenmann, "Industrial applications of computed tomography", Man. Techn., 63 (2) (2014), pp. 655-677

[5] C. Ziehm, S. Hantscher, J. Hinken, C. Ziep, M. Richter, ,Near field focusing for nondestructive microwave testing at $24 \mathrm{GHz}$ - Theory and experimental verification", Elsevier, Case Studies in Nondestructive Testing and Evaluation, vol. 6, pp. 70-78, Nov. 2016

[6] D. Beilken, J. Hinken, "Microwave Based Nondestructive Testing Using Modified Eddy Current Systems", European Conference on Non-Destructive Testing, 2016

[7] S. Kharkovsky and R. Zoughi, "Microwave and millimeter wave nondestructive testing and evaluation - Overview and recent advances", IEEE Instrumen-tation and Measurement Magazine, vol. 10, no. 2, pp. 26-38, Apr. 2007, doi: 10.1109/MIM.2007.364985

[8] C. Krebs, J. Rubart, A. Hommes, R. Brauns, D. Nüssler, „High resolution measurements to determine the permittivity in artificial structures", International Conference on Millimeter Waves, Rome, Italy, 2010, doi: 10.1109/ICIMW.2010.5613004

[9] D. Nuessler, M. Schubert, S. Kose, N. Pohl, "Swifter Security Scanning", IEEE Microwave Magazine, vol. 18, no. 6, Sept./Oct. 2017, doi: 10.1109/MMM.2017.2712018
[10] F. Ellrich, T. Weinland, J. Klier, J. Jonuscheit, and R. Beigang, "Terahertz Waves - A New Spectral Band for Industrial Measurement Techniques“, Technisches Messen, vol. 77, no. 9, pp. 452-461, 2010

[11] G. von Freymann, J. Jonuscheit, " $\mathrm{THz}$ technology - from hype to market”, Photonik. Sonderheft LASER + PHOTONICS, pp. 102105, 2016

[12] B. Baccouche, S. Mohammadzadeh, A. Keil, M. Kahl, P. Haring Bolivar, T. Loeffler, J. Jonuscheit, W. Sauer-Greff, R. Urbansky, F. Friederich, "Illumination aspects of sparse line arrays for 3D terahertz imaging", 41st Int. Conference on Infrared, Millimeter, and Terahertz Waves, IRMMW-THz 2016, doi: 10.1109/IRMMW-THz.2016.7758825

[13] M. Nezadal, J. Schur, L. Schmidt, "Nondestructive testing of glass fibre reinforced plastics with a synthetic aperture radar in the lower THz region", International Conference on Infrared, Millimeter and Te-rahertz waves, Kaiserslautern, 2012, doi: 10.1109/IRMMWTHz.2012.6380301

[14] R. Wegerhoff, O. Weidlich, M. Kässens. „Basics of Light Microscopy \& Imaging", 18-19 (2011), GIT Verlag: Imaging \& Microskopy

[15] Nesterov, M.; et.al.: „Evaluation of Different Antenna and Data Analysis Concepts for Microwave Based Non-Destructive Testing", 12th European Conference on Non-Destructive Testing (ECNDT 2018), 11.-15.06.2018, Göteburg, Schweden, 2018.

[16] G. D. Boreman, „Modulation Transfer Function in Optical and Electro-Optical Systems", Bellingham, Washington: SPIE-The International Society for Potical Engineering, 2001, S. 69-76, ISBN: 0-8194-4143-0

[17] Eva-Maria Brinkmann, „Dekonvolution in der Mikroskopie", Bachelorarbeit 2012, Westfälische Wilhelms-Universität Münster

[18] P. Sarder, A. Nehorai, "Deconvolution methods for 3-d fluorescence microscopy images", IEEE Signal Processing Magazine, S. 32-45, Mai 2006, doi: 10.1109/MSP.2006.1628876

\section{Kontakt}

Thomas Gagelmann: thomas.gagelmann@ifak.eu 